\title{
DIABOT-An Expert System in management of Diabetics using Artificial Intelligence
}

\author{
Sanyukta Suman \\ B.E in CSE, BMSITM \\ Bangalore, India
}

\author{
Srivani P. Badala \\ Assistant Professor, BMSITM \\ Bangalore, India
}

\author{
Dibya Malla Thakuri \\ B.E in CSE, BMSITM \\ Bangalore, India
}

\author{
Akshay Raj Manandhar \\ B.E in CSE, BMSITM \\ Bangalore, India
}

\author{
Riyanna Luvella Martis \\ B.E in CSE, BMSITM \\ Bangalore, India
}

\begin{abstract}
The proposed project, "Diabot" is targeted towards those who are diabetic, to be able to take care of themselves without having the trouble to arrange for appointments or consultation. The debate is an artificial intelligence based expert system that can be used by diabetic patients for regulating and examining their current vital information at the convenience of their own homes. This boat is, therefore a "companion" both for the patient, which can be accessed at any time. Diabot will measure the necessary parameters of the patient's blood and will consult them about their medication or suggest activities to do according to the reading that is taken by the reading device. The "Diabot" will have a knowledge base through various medical databases and case files pertaining to diabetic patients, which will allow the system to make inferences for making decisions.

The main objective of the proposed project is to design an expert system for diagnosis of diabetes and to provide the patient with an equivalent consultancy as such provided by a doctor, so the patient has easy access to health care. From this proposed project, there can be a hope to provide a costeffective and user-friendly personal healthcare bot which is an expert system in the management of diabetes using Artificial Intelligence.
\end{abstract}

\section{Keywords}

Artificial Intelligence, Expert System, Knowledge Base, Inference Engine, Diabetes

\section{INTRODUCTION}

Expert systems are intended to take care of complex issues by thinking through forms of learning, signified mainly as ifthen rules than through predictable procedural code. A specialist framework is separated into two subsystems: the knowledge base and inference engine. The knowledge base determines the truths and rules. The inference engine smears the rules to the identified facts to deduce it into new facts. The Diabot is an artificial intelligence expert system that can be utilized by diabetic patients for regulating and examining their current vital information at the convenience of their home. Diabetes, a prolonged disease, requires steady medical care and self-administration, instruction for patients to stay away from intense confusions and to lessen the danger of difficulties at the long haul. The most common form of diabetes originates from a progressive insulin secretion defect where the body does not produce adequate insulin where the insulin produced by blood is not sufficient for the body. Expert systems are computerized algorithms that take care of issues in a non-procedural way utilizing information from human specialists to reproduce human thinking and reasoning.
They are also called knowledge-based systems or inferencebased programs. This proposed project is a health care, rulebased expert system for diabetics using artificial intelligence.

\section{RELATED WORK}

A review of the related work is being highlighted to understand the key elements of the existing system. The author [1] combines fuzzy logic and an ontology system to build a patient-centered treatment decision support system which can infer the individualization $\mathrm{HbAlc}$ target and recommend anti-diabetic drugs for patients with T2DM which also serve as a guide for specialty doctors. However, it fails to consider all the parameters for the diagnosis and suggestions. The authors [2] have explained how AI can be a dietitian to an individual and can prevent diabetes. People have a different lifestyle and the impacts of the same events are different for different people so by making it personalized better results can be obtained. On the other hand a comprehensive study of medical expert systems for diagnosis of various diseases using Fuzzy Logic [3], [4]. The Knowledge Base encloses information with reference to diseases which are characterized as a set of if-then production rules. One of the drawbacks is, even though covers diagnosis of various diseases, it is limited to a general perspective of each disease; it doesn't cover specifics. Another work by authors [5] presents an application of "Automatic Multilayer Perceptron (AutoMLP)" which is combined with an outlier detection method Enhanced Class Outlier Detection using distance-based algorithm to create a novel prediction framework. This paper [6] reveals a designed system that is a rule-based expert system, for knowledge representing some rules has been used. The structure of these rules is IF-THEN. IF is demonstrating the situation and THEN shows the suggestion. The existing systems do not provide the complete optimal solution. Most of these works have been concentrated to design a model for generic predictions and are with poor performances.

The main objective of this proposed project is to overcome the limitations found in a few of the existing systems and to design an expert system to monitor important metrics like heart rate, blood glucose level, body temperature, blood pressure, etc. in the diabetic patient and to provide the patient with an equivalent consultancy such as any provided by a doctor, so the patient has easy access to the healthcare. With this proposed project management of diabetes can be personalized, customized and gives every patient to monitor his sugar level. This project also aids to be smart by providing decision support system.

The Table 1 shown below shows the existing apps that assist in the management of diabetes. 
Table 1: Existing Apps and its applications

\begin{tabular}{|c|c|}
\hline Name & Application \\
\hline GLOOKO & $\begin{array}{l}\text { As specified in [7], Glooko tracks } \\
\text { the individual's medical usage, carb } \\
\text { counts, and lifestyle info. }\end{array}$ \\
\hline MYNETDIARY & $\begin{array}{l}\text { In [8], it is specified how } \\
\text { mynetdiary helps to manage not } \\
\text { only type } 1 \text { and } 2 \text { diabetes, but also } \\
\text { pre-diabetes and gestational } \\
\text { diabetes through the built-in GPS } \\
\text { and the foods via the built-in } \\
\text { barcode scanner. }\end{array}$ \\
\hline $\begin{array}{l}\text { DIABETES IN } \\
\text { CHECK }\end{array}$ & $\begin{array}{l}\text { Focuses on proactive eating and } \\
\text { fitness [9]. It provides a reference } \\
\text { guide for the best foods to eat as } \\
\text { well as diabetes-friendly recipes } \\
\text { and even a personalized meal plan. }\end{array}$ \\
\hline BG MONITOR & $\begin{array}{l}\text { In [10] the author provides an easy } \\
\text { input system and calculates insulin } \\
\text { levels, clearly can set up the } \\
\text { person's blood glucose targets and } \\
\text { add multiple types of insulin to take } \\
\text { the right medicines and amounts at } \\
\text { the right times. }\end{array}$ \\
\hline DARIO & $\begin{array}{l}\text { In [11], it includes a simple-to-use } \\
\text { glucose meter, a disposable test } \\
\text { strip cartridge holding } 25 \text { test strips, } \\
\text { and lancing device. }\end{array}$ \\
\hline GLUCOSIO & $\begin{array}{l}\text { In [12], it tracks important metrics } \\
\text { like A1C, body weight, ketones, } \\
\text { cholesterol, blood pressure, and } \\
\text { more. }\end{array}$ \\
\hline
\end{tabular}

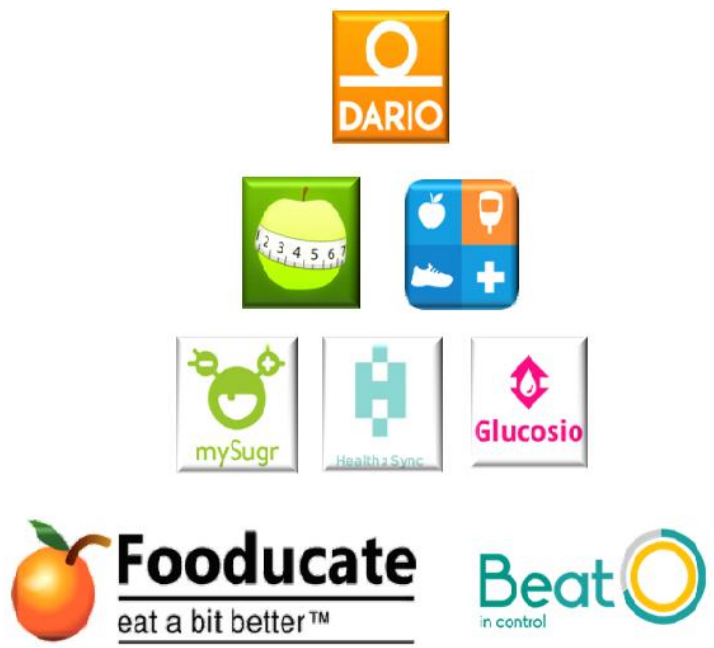

Figure 1: Existing Apps related to Diabetes Management System

\section{SYSTEM DESIGN}

The proposed architecture as shown in Figure 2 consists of three main modules. These modules are the user interface, the hardware \& sensors and the expert system.

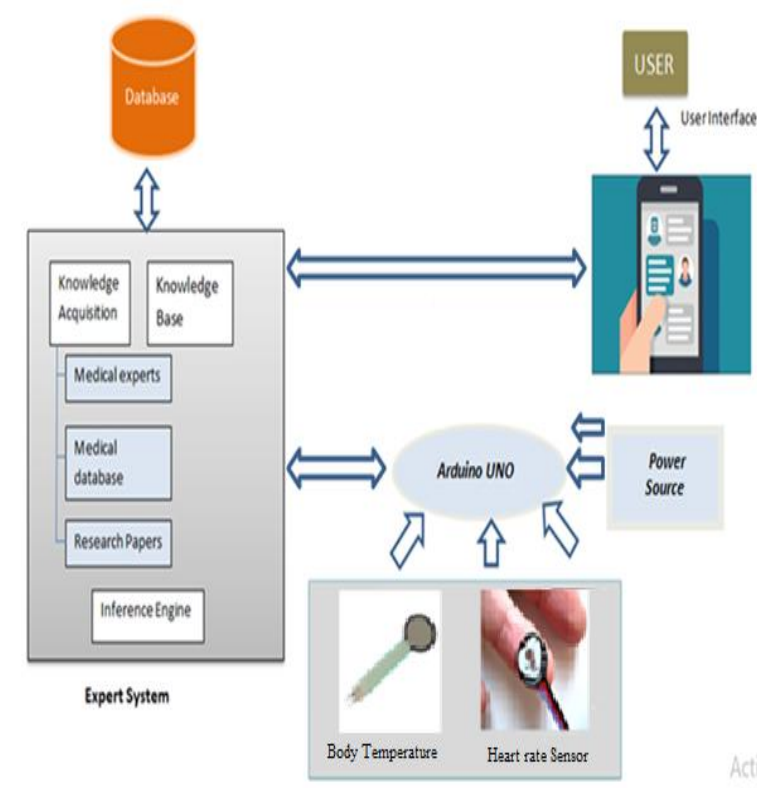

Figure 2: General Architecture of Diabot

The user interface consists of the document and interface through which the user will enter their information and it will record the data given by the user. The information it will ask for being basic such as the user's family history, health complications, symptoms faced by the user, etc. This module will also display to the user the output derived by the expert system.

The second module is designed with a combination of hardware and sensors that are integrated with Arduino UNO. This microcontroller is embedded with various sensors such as pulse rate, body temperature. This physical module will be used to take the user's regulatory statistics and will be fed to the expert system. Apart from sensory inputs the user should manually provide the glucose level input to the expert system which is a major concern in diabetes management systems. The expert system is then initiated to interpret the readings.

The third module which is an Expert system consists of two sub-modules: Inference Engine and Knowledge base. The Inference Engine is designed using PHP and MYSQL which will refer to the Knowledge-base that is created using SWIProlog. The Inference engine will infer a diagnosis from the knowledge base that has been created using various databases and also a personal database that keeps track of the user's health records. The inferred result is then also used to help predict any trends viewed by the expert system and is used to give a thorough feedback for the user.

\subsection{Data Flow}

The flow chart of the expert system shown in Figure 3 demonstrates the process of the system that is being designed. Initially, the user gives inputs like his personal information like age, height, weight, etc. Apart from these inputs, the user also feeds various health-related data into the system based on the queries asked by the expert system. When this process completes, the data is stored in a database.

The inference engine then compares the received data to the standard level of all the health metrics present in the knowledge base. To start with analysis, the system analyzes the data from sensory inputs and then starts with the diagnosis. The symptoms are classified and identified to find 
the root cause of the problem. Then, various diagnostic measures are suggested to the user which will help the user keep diabetics on track and live a healthy life.

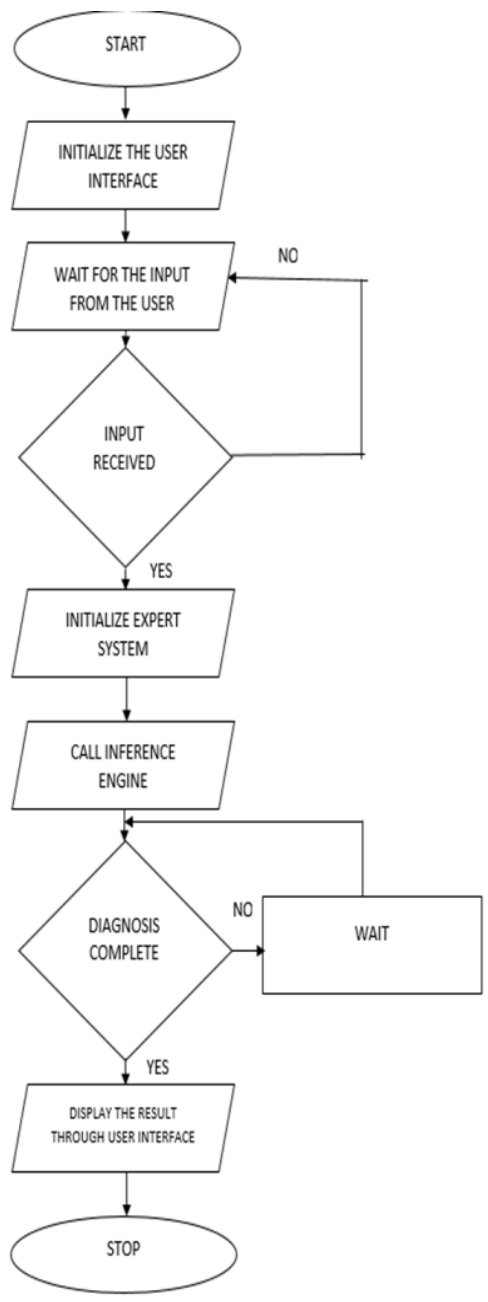

Figure 3: The data flow of Diabot

\section{IMPLEMENTATION AND RESULTS}

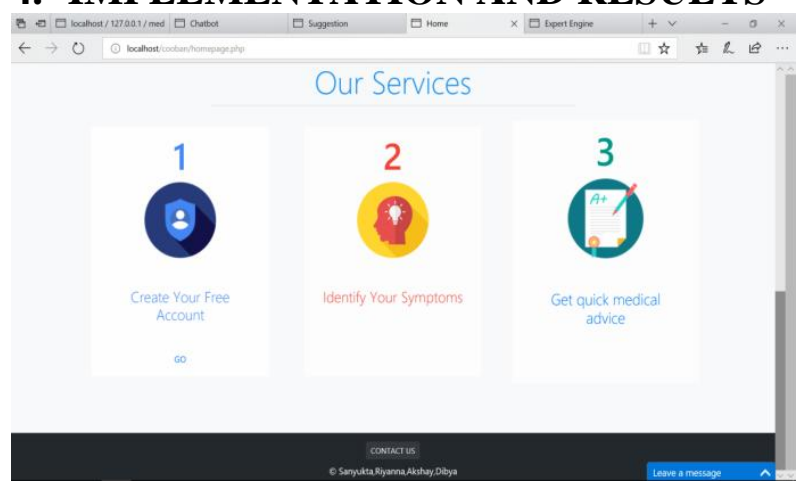

Figure 4: Home Screen of Diabot

Figure 4 is a snapshot of Homepage of the system, through which we can navigate to expert engine page, Chabot page or suggestion page.

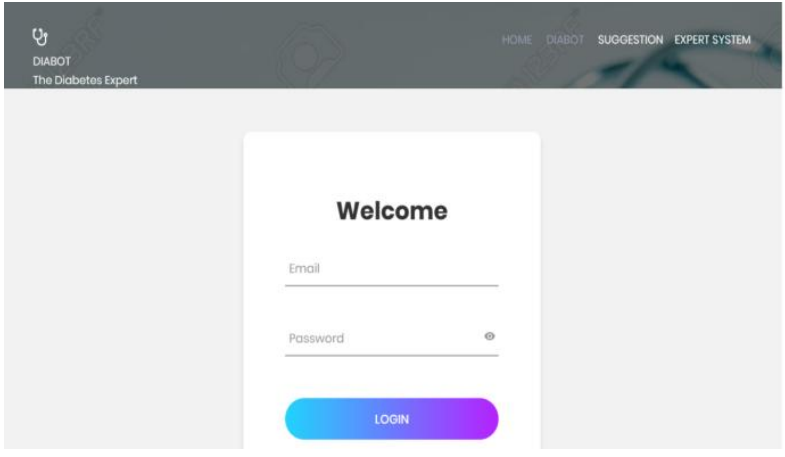

Figure 5: Login Page

Figure 5 is a snapshot of Login Page of the Expert Engine where the user can register and login into the expert system. Once he navigates and enters into the main page to identify the symptoms based or get expert based advice through a set of a questionnaire from the knowledge base. In this module, the user can select options for each question. Here, questions include parameters such as gender, blood pressure count, glucose level count, family history, body mass index, etc., which are the parameters to be considered in identifying the type of diabetes.

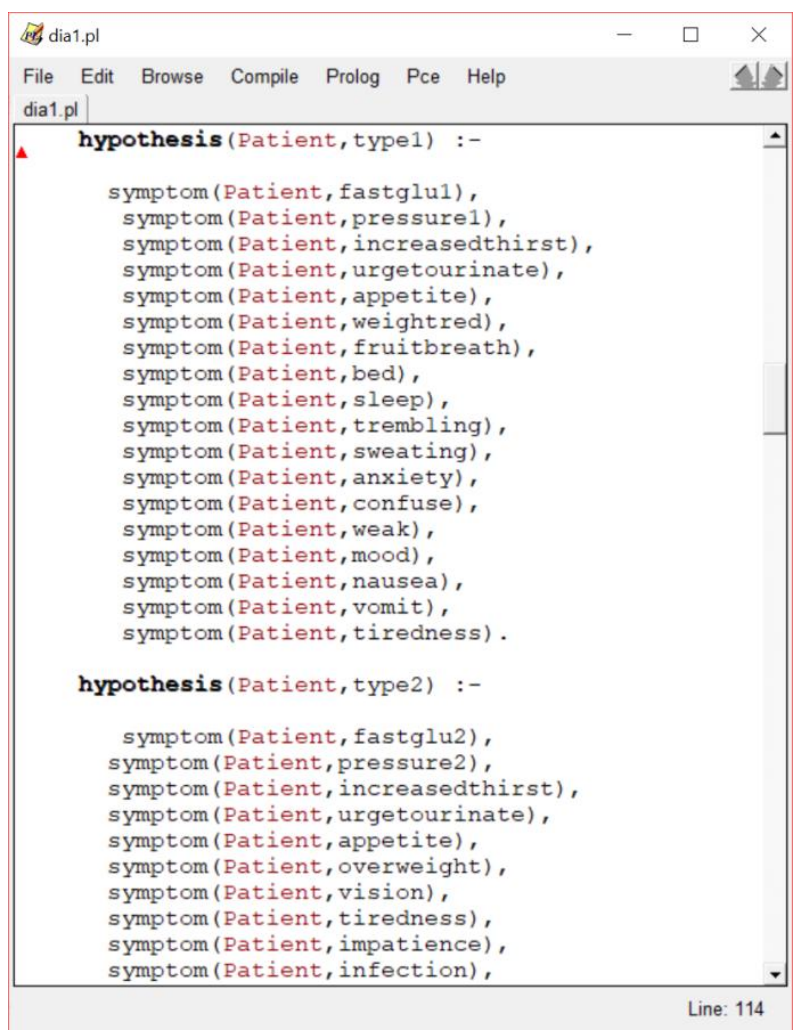

Figure 5: Symptoms designed on the Knowledge Base

Figure 5 is a snapshot of a sample of Knowledge Base of the system. There are separate hypothesis for each type of diabetes, the symptoms are mentioned, according to the type of diabetes, when the user gives input to the expert engine, it compares the input with data in the knowledge base and infers output according to the hypothesis.

With the integration of pulse rate sensor, temperature sensor and glucose monitoring system, this knowledge based expert system could analyze the hazardous and unpredictable fluctuations in the body which is due to the aspects like an improper diet plan, stress level and no physical activities. The 
system also suggests the particular patient explore the ways to control and manage his diabetes through diet plans, physical activities, health tips and many more.

The system monitors the patient's health status and helps the patient to know his condition, predict and prevent any anomalies. This system is an effective tool for improving the patient's quality of life.

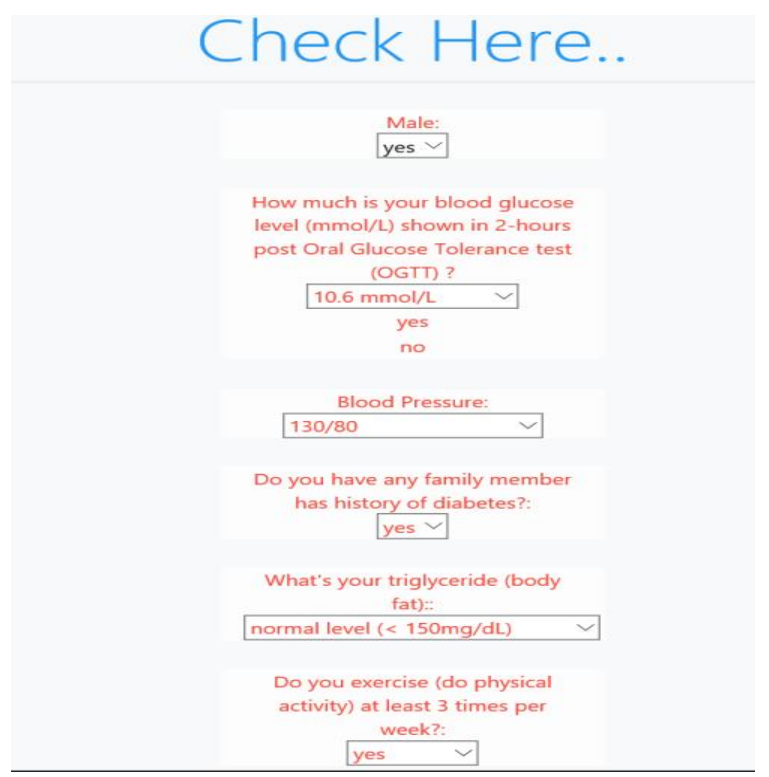

Figure 6: Sample questionnaire from the Knowledge Base

Based on the questionnaire shown in Figure 6, further the expert system assists you with the required diet plan, physical activity plan.

Based on the answers given by the user, the experts system navigates to the suggestion page, to assist the patient with proper diet plan and physical activity. The module also detects the category of diabetes the patient is suffering from.

A new study suggests that the person can get a sense of his diabetes risk by counting his heartbeats. The normal resting heart rate is between 60-100 Beats per Minute (BPM). While most of us have a resting heart rate of 65-85 BPM, those who have a resting heart rate of over 85 BPM have a higher chance of developing diabetes.

\section{CONCLUSION}

Diabot can be utilized by a wide range of people who are suffering from Type-1 and Type-2 diabetes. In addition, for the diabetic patients living in a rural area, this device can be a great help for them as they can benefit from the health care provided which otherwise they would not receive. The proposed project will also keep track of the patient's personal habit, count the calorie intake as well, thus can act as a personalize health assistant. By using Diabot, the user can get answers to their queries and identify the type of diabetes they might have. The user can check for suggestions provided by the expert system, subsequently maintaining their health. The patient can check their heart rate and body temperature. Diabot is a reliable healthcare, bot which will be very useful for diabetic patients.

\section{FUTURE ENHANCEMENT}

The use of Fuzzy logic can be implemented in the system which imitates the way of human reasoning and decisionmaking process. It includes all the intermediate possibilities like certainly yes, maybe, possibly no, certainly not etc. that exists between a digital Yes and No i.e. real numbers between 0 and 1, unlike typical computers. Fuzzy logic will help to deal with uncertainty in this project. It may not give accurate reasoning, but it may give acceptable reasoning. This project can be enhanced in the future by integrating more sensors in this proposed project, like Blood Pressure sensor, inbuilt Glucose meter sensor, etc. By including more sensors, this project will be able to take more parameters from the patient and as a result, it can produce more accurate results. Additionally, we can include the use of decision trees for classification and structuring process. This helps to simplify the complexity of the system which increases with the addition of new information to the expert system.

\section{ACKNOWLEDGMENTS}

Thanks to the Guide, Co-ordinators for making this project successful.

\section{REFERENCES}

[1] Chen, Rung-Ching, et al. "A decision support system for diabetes medicine selection using patient centered treatment based on fuzzy logic and domain ontology." International Journal of Innovative Computing, Information and Control 13.5 (2017): 1681-1692.

[2] Vachon, Brigitte, Ai-Thuy Huynh, Mylaine Breton, Louise Quesnel, Michel Camirand, Jeannette Leblanc, and Sylvie Tardif. "Patients' expectations and solutions for improving primary diabetes care." International journal of health care quality assurance 30 , no. 6 (2017): 554-567.

[3] Singla, Jimmy, Dinesh Grover, and Abhinav Bhandari. "Medical expert systems for diagnosis of various diseases." International Journal of Computer Applications 93, no.7 (2014).

[4] Lalka, Neeru, and Sushma Jain. "Fuzzy based expert system for diabetes diagnosis and insulin dosage control." In Computing, Communication \& Automation (ICCCA), 2015 International Conference on, pp. 262267. IEEE, 2015.

[5] Nawaz, R., M. Jahangir, M. Ahmed, and H. Afzal. "An expert system for diabetes prediction using auto tuned multi-layer perceptron." (2017).

[6] Zeki, Tawfik Saeed, Mohammad V. Malakooti, Yousef Ataeipoor, and S. Talayeh Tabibi. "An expert system for diabetes diagnosis." American Academic \& Scholarly Research Journal 4, no. 5 (2016): 1.

[7] Available [Online] from "Glooko" https://www.myomnipod.com/poddersupport/aps-datamanagement/glooko.

[8] Available [Online] from https://www.mynetdiary.com/diabetes.html

[9] Available [Online]: Diabetes in check: http://www.diabetesincontrol.com/diabetes-in-checkblood-glucose-carb-tracker/

[10] Available [Online]: from June 2018:The best apps: https://www.healthline.com/health/diabetes/top-iphoneandroid-apps

[11] Availabale [Online]: From June 2018: My dario: https://mydario.com/the-app/

[12] Available(online) from : https://www.glucosio.org/ 